\title{
Oscillatory Turing Patterns in a Simple Reaction-Diffusion System
}

\author{
Ruey-Tarng LiU* and Sy-Sang LiaW \\ Department of Physics, National Chung-Hsing University, 250 Guo-Kuang Road, Taichung, Taiwan \\ Philip K. MAINI \\ Centre for Mathematical Biology, Mathematics Institute, \\ Oxford University, 24-29 St. Giles', Oxford OX1 3LB, U.K.
}

(Received 15 August 2006)

\begin{abstract}
Turing suggested that, under certain conditions, chemicals can react and diffuse in such a way as to produce steady-state inhomogeneous spatial patterns of chemical concentrations. We consider a simple two-variable reaction-diffusion system and find there is a spatio-temporally oscillating solution (STOS) in parameter regions where linear analysis predicts a pure Turing instability and no Hopf instability. We compute the boundary of the STOS and spatially non-uniform solution (SSNS) regions and investigate what features control its behavior.
\end{abstract}

PACS numbers: 82.40.Ck, 87.15.Aa, 47.54.-r

Keywords: Oscillatory Turing pattern, Reaction-diffusion, Nonlinear

\section{INTRODUCTION}

In 1952, Turing proposed [1] that chemicals called morphogens diffusing in space could interact to form stable spatially non-uniform distributions. The concept is contradictory to the common intuitive understanding of the effect of diffusion. A reaction-diffusion system takes the following form:

$$
\frac{\partial M}{\partial t}=F(M)+D \nabla^{2} M,
$$

where $M$ stands for the concentration vector of morphogens, $D$ is the diagonalized matrix of positive diffusion constants, and $F(M)$ contains the reaction kinetics of the system. When there is no diffusion $(D=0)$, $F(M)$ is such that $\mathrm{M}$ will reach a uniform stable distribution. For non-zero diffusion, under specific conditions, an instability of the uniform distribution can be induced (a phenomenon known as "diffusion-driven" instability or "Turing" instability), which then will grow exponentially with time, only to be bounded finally by the nonlinear terms in $F(M)$ and form a stable spatially nonuniform solution (SSNS). There have been many Turingtype models used for generating patterns with applications to mammals [2-4], fish [3,5-7], ladybugs [8], bacterial colonies [9-11], and phyllotaxis [12].

Other types of patterns have also been found in reaction-diffusion systems. Among them, spatiotemporally oscillating solutions (STOS) have attracted

\footnotetext{
*E-mail: rtliu@phys.nchu.edu.tw
}

much attention $[13,14]$. The STOS is different from the well-known uniform Hopf oscillation and is generally believed to be due to an interaction of the Turing instability with either a Hopf or a wave instability [15-20]. Recently, Vanag and Epstein [21] found an out-of-phase STOS in a bistable reaction-diffusion system with no presence of a Hopf or wave instability. In this report, we consider a simple reaction-diffusion system which has only one steady state. We found that, without interaction with either a Hopf or a wave instability, the Turing instability, together with the effects of a non-linear interaction in a two morphogen system, can yield both SSNS and STOS.

\section{MODEL AND LINEAR ANALYSIS}

We study a simple reaction diffusion system characterized by the equation for two distributions $u$ and $v$ [22]:

$$
\begin{aligned}
& \frac{\partial u}{\partial t}=D \delta \nabla^{2} u+\alpha u+v-\alpha r_{3} u v^{2}-r_{2} u v \\
& \frac{\partial v}{\partial t}=\delta \nabla^{2} v-\alpha u+\beta v+\alpha r_{3} u v^{2}+r_{2} u v .
\end{aligned}
$$

Note that in this model, $u$ and $v$ should be thought of not as morphogen concentrations, but as deviations from some non-zero (positive) fixed concentration profile. In this case, negative values of $u$ and/or $v$ are physically realistic. The nonlinear reaction between $u$ and $v$ is given by the sum of a quadratic term and a cubic term with negative coefficients being $-r_{2}$ and $-\alpha r_{3}$, respectively. 
(a)
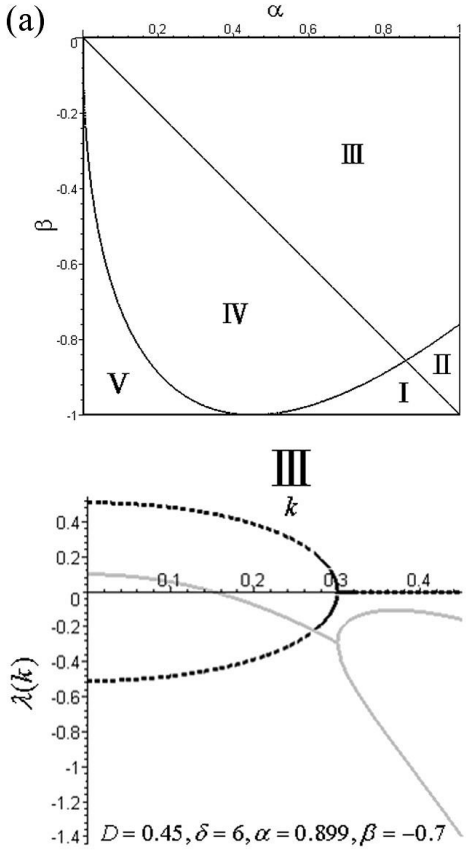

(b)
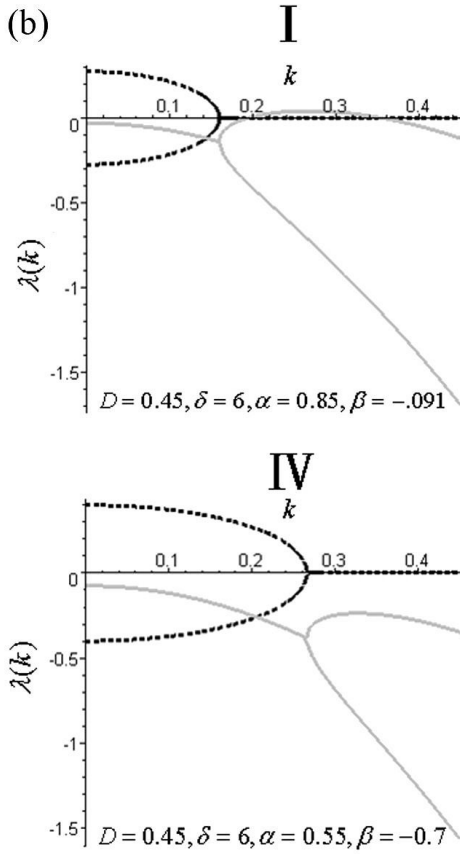
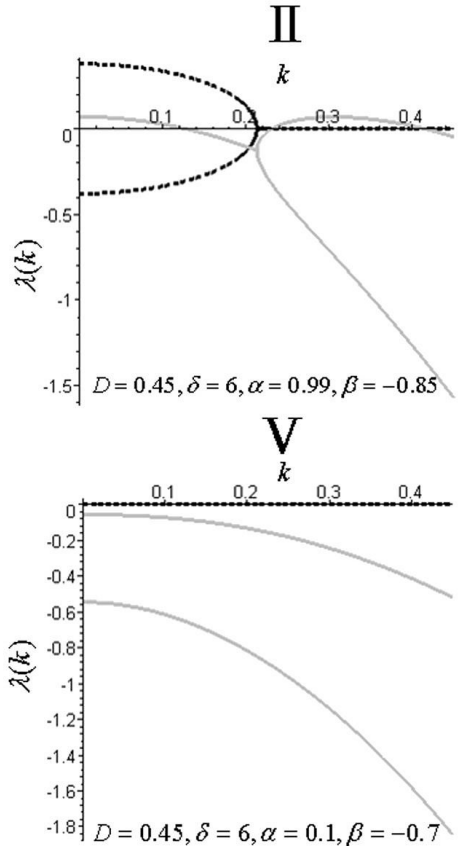

Fig. 1. (a) Two bifurcation lines separate the parameter space into five domains: (I) Turing unstable; (II) Turing-Hopf unstable; (III) Hopf unstable; (IV) and (V) stable. (b) Dispersion relations of the five domains. The real and the imaginary parts of the eigenvalue are shown by gray solid lines and black dotted lines, respectively.

The origin $(0,0)$ is the only spatially uniform steady state, and the following conditions must be satisfied for a diffusion-driven instability $[22,23]$ :

$$
\begin{aligned}
& \alpha+\beta<0 \\
& \alpha(\beta+1)>0 \\
& D \beta+\alpha>0 \\
& (D \beta+\alpha)^{2}-4 D \alpha(\beta+1)>0 .
\end{aligned}
$$

In the standard way, we assume that $u$ and $v$ take the form

$$
u(\vec{x}, t) \sim u_{0} e^{\lambda t} e^{i \vec{k} \cdot \vec{x}} \text { and } v(\vec{x}, t) \sim v_{0} e^{\lambda t} e^{i \vec{k} \cdot \vec{x}}
$$

in the linearized version of Eq. (2), yielding a dispersion relation from which one can choose parameters to allow only some of the modes with $\operatorname{Re}(\lambda)>0$ to grow in time. The dispersion relation $\lambda(k)$ relating the temporal growth rate to the spatial wave number can be found from the characteristic equation

$$
\begin{gathered}
\lambda^{2}+\left[(1+D) \delta k^{2}-\alpha-\beta\right] \lambda+D \delta^{2} k^{4} \\
-\delta(\alpha+D \beta) k^{2}+\alpha(\beta+1)=0 .
\end{gathered}
$$

The bifurcation diagram is shown in Fig. 1. The Hopf bifurcation line $(\operatorname{Im}(\lambda) \neq 0, \operatorname{Re}(\lambda)=0$ at $k=0)$ and the Turing bifurcation line $\left(\operatorname{Im}(\lambda)=0, \operatorname{Re}(\lambda) \neq 0\right.$ at $k=k_{T}$ $\neq 0)$ separate the parametric space into five distinct domains. In domains IV and V, the steady state is the only spatially uniform stable solution of the system. Domains I and III are regions in which this steady state solution is Turing and Hopf unstable, respectively. In domain II, Turing and Hopf instabilities coexist.

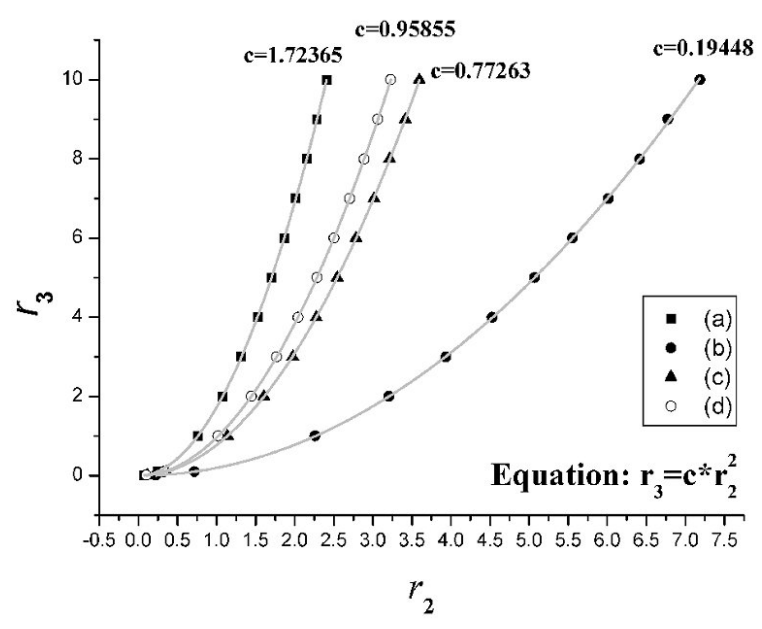

Fig. 2. Boundaries (fitted curves) of the SSNS and the STOS phases are found by scanning $r_{2}$ for each fixed $r_{3}$. Only SSNS is found on the left of each curve, and STOS exists on the right. Curves (a) and (b) have the same parameters of $D=0.516, \delta=4, \alpha=0.92, \beta=-0.965$, but with different random initial distributions for $u$ and $v$. Curve (c) has the same initial distributions as (b), but with parameters $D=$ $0.39, \delta=4, \alpha=0.88$, and $\beta=-0.91$. The values of $\alpha$ and $\beta$ for curves (a), (b), and (c) are in the pure Turing unstable domain while those for curve (d) are in the Turing-Hopf unstable domain, $D=0.45, \delta=6, \alpha=0.95, \beta=-0.91$.

\section{SIMULATION RESULTS}

It was known previously that to produce an "oscillatory Turing" pattern one has to excite the Turing-Hopf 

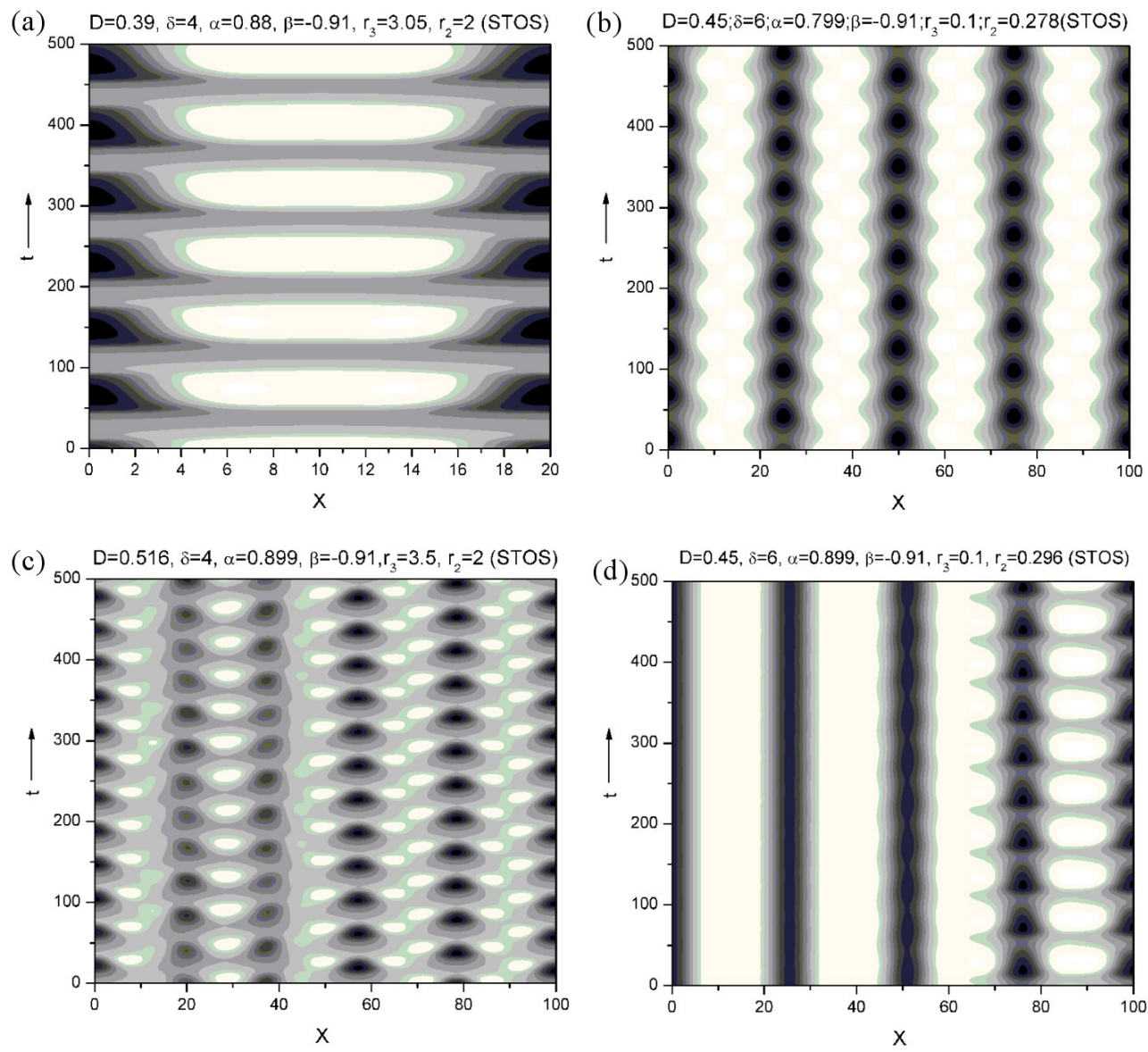

Fig. 3. Concentration of $u$ shown in a space-time plot. The concentration profile of $v$ (not shown) follows that of $u$. Four different kinds of patterns are found in the Turing unstable domain when the quadratic effect is strong: (a) in-phase oscillatory patterns; (b) out-of-phase oscillatory patterns; (c) mixture of in-phase and out-of-phase oscillatory patterns; (d) combination of Turing and oscillatory patterns.

or Turing-wave instabilities [19]. We, thus, chose the values of the linear parameters $\alpha$ and $\beta$ to be within domain II of Fig. 1(a) where Turing and Hopf instabilities coexist. We found there were indeed SSNS for some parameter sets of $\left(r_{2}, r_{3}\right)$ and STOS for others. While fixing the parameters $D$ and $\delta$, and initial random distributions for $u$ and $v$, we scanned the non-linear parameters $r_{2}$ and $r_{3}$. We found that the $\left(r_{2}, r_{3}\right)$ parameter space could be separated into two regions by a curve of the form $r_{3}=$ $c r_{2}^{2}$ (Fig. 2). Only the SSNS is found in the region $r_{3}>$ $c r_{2}^{2}$, and STOS exists in the region $r_{3}<c r_{2}^{2}$. This result suggests that increasing the strength of the quadratic term can induce STOS.

Surprisingly, even for parameters $\alpha$ and $\beta$ within domain I of Fig. 1(a), where only the pure Turing instability exists, we obtain STOS, as well when $r_{2}^{2} / r_{3}$ exceeds a certain critical value. The critical value depends on the parameters $\alpha, \beta, \delta$, and $D$, and on the initial distributions of $u$ and $v$ (Fig. 2). Note that there is no Hopf instability for the parameters $\alpha$ and $\beta$ that we used. This means that a Hopf instability is not necessary for generating STOS. In the pure Turing instability region, STOS can be excited by a suitable non-linear interaction.

We have found four types of oscillatory Turing patterns in both one- and two-dimensional space when $r_{2}^{2} / r_{3}$ is larger than its critical value. They are in-phase oscillatory patterns (Fig. 3(a)), out-of-phase oscillatory patterns (Fig. 3(b)), a mixture of in-phase and out-ofphase oscillatory patterns (Fig. 3(c)), and a combination of Turing and oscillatory patterns (Fig. 3(d)).

\section{EFFECTS OF THE NON-LINEAR REACTION}

We can understand the emergence of STOS qualitatively by looking into Eq. (2) more carefully. If there are no non-linear interaction terms, namely, $r_{2}=r_{3}=0$, a random perturbation will cause the distributions of $u$ and $v$ to grow exponentially. There would be no SSNS or STOS. Note the cubic term $-\alpha r_{3} u v^{2}$ in the equation for $u$ always counters the growth of $u$ while the quadratic term $-r_{2} u v$ can enhance the growth of $u$ if $u$ and $v$ have 


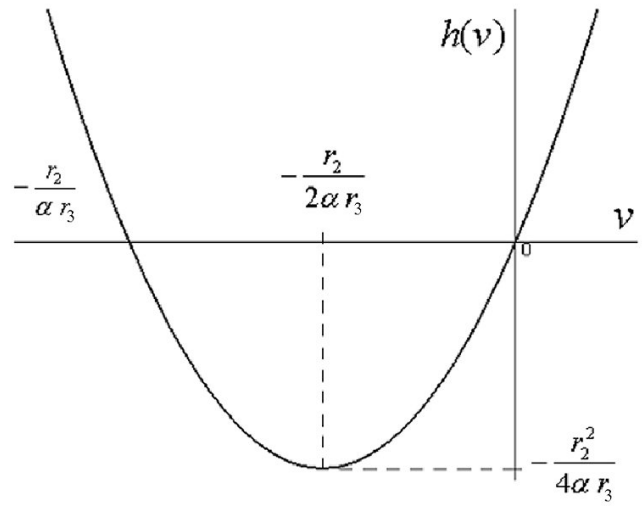

Fig. 4. The function $h(v)$ (see text) has a negative value when $v$ is between $\frac{-r^{2}}{\alpha r_{3}}$ and 0 and has a minimal value $-\frac{r_{2}^{2}}{4 \alpha r_{3}}$. The negativity of $h(v)$ will enhance the instability of the system characterized by Eq. (2).

opposite signs. Thus, the system will still diverge if the quadratic term alone is included in the equation. On the other hand, adding the cubic term would "rein in" the system into the SSNS. If both terms are present, the system will go from the SSNS to STOS when we increase the strength of the quadratic term. Let us make a scale transformation for the distributions $u$ and $v: u \rightarrow s u$ and $v \rightarrow s v$. Eq. (2) will be

$$
\begin{aligned}
& \frac{\partial u}{\partial t}=D \delta \nabla^{2} u+\alpha u+v-s^{2} \alpha r_{3} u v^{2}-s r_{2} u v \\
& \frac{\partial v}{\partial t}=\delta \nabla^{2} v-\alpha u+\beta v+s^{2} \alpha r_{3} u v^{2}+s r_{2} u v .
\end{aligned}
$$

We see that the ratio of the square of the coefficient of the quadratic term to the absolute value of the coefficient of the cubic term is independent of the scale $s$. This scale-free ratio, which is given by $r_{2}^{2} / \alpha r_{3}$, is then the significant parameter that one can use to indicate the relative strength between the quadratic and cubic terms. This explains what we found in Sec. III, namely that the system will lead to STOS when $r_{2}^{2} / r_{3}$ exceeds a certain critical value (with a given $\alpha$ ).

If we rewrite the non-linear term in the equation for $u$ as $-u\left(\alpha r_{3} v^{2}+r_{2} v\right) \equiv-u h(v)$, we find that $h(v)$ has a negative value when $\mathrm{v}$ is between $-r_{2} /\left(\alpha r_{3}\right)$ and 0 and its minimal value is $-r_{2}^{2} /\left(4 \alpha r_{3}\right)$ (Fig. 4$)$. The negativity of $h(v)$ will induce an instability of the sytem, as explained above. If we modify the original system by subtracting a linear term $\left(r_{2}^{2} / 4 \alpha r_{3}\right) u$ from the right-hand side of the upper equation of Eq. (2) and adding the same term to the right-hand side of the lower equation of Eq. (2), to compensate for the instability effect caused by the nonlinear term, then only the SSNS exists and STOS will not occur. Our simulations confirm this. The suppression of STOS by changing the linear terms of the equation can also be understood from the change in the Turing space. The added linear term has effectively changed the key parameter $\alpha$ to $\alpha^{\prime}=\alpha-r_{2}^{2} /\left(4 \alpha r_{3}\right)$ which, as can be seen from Fig. 1(a), moves the system from the Turing unstable region (domain I in Fig. 1 (a)) to a stable region (domain IV) where no STOS is possible.

\section{CONCLUSION}

It is generally believed that there should be no Turing oscillatory patterns in the domain of pure Turing instability. According to the standard linear analysis, if a system is Turing unstable, its sole singularity has to be an unstable saddle point; thus, no limit cycle is possible. The pure Turing instability does not interact with other instabilities, such as the Turing-Hopf interaction reported previously, to generate oscillatory patterns.

In this research, we, nevertheless, found that nonlinear effects could induce oscillatory patterns in the pure Turing unstable domain. For our system, the cubic and the quadratic terms have opposite effects on the solution behavior. The former tends to bring the system to a stable pattern while the latter increases the instability of the system. The final patterns result from their competition. Only stable Turing patterns are possible if the quadratic term is missing. When the ratio of the square of the coefficient of the quadratic term to the absolute value of the coefficient of the cubic term is larger than a critical value, oscillatory Turing patterns emerge. The critical value depends on the diffusion constants, the linear parameters, and the initial distributions.

\section{ACKNOWLEDGMENTS}

This work is supported by grants from the National Science Council under grant number NSC95-2112-M005008 and from the National Center for Theoretical Sciences of Taiwan.

\section{REFERENCES}

[1] A. Turing, Phil. Trans. R. Soc. London B 237, 37 (1952).

[2] J. B. L. Bard, J. Theor. Biol. 93, 363 (1981).

[3] J. D. Murray, Philos. Trans. R. Soc. Lond B Biol. Sci. 295, 473 (1981); J. Theor. Biol. 88, 161 (1981).

[4] R. T. Liu, S. S. Liaw and P. K. Maini, Phys. Rev. E 74, 011914 (2006).

[5] S. Kondo and R. Asai, Nature 376, 765 (1995).

[6] T. Höfer and P. K. Maini, Nature 380, 678 (1996).

[7] C. Varea, J. L. Aragón and R. A. Barrio, Phys. Rev. E 56, 1250 (1997).

[8] S. S. Liaw, C. C. Yang, R. T. Liu and J. T. Hong, Phys. Rev. E 64, 041909 (2001).

[9] E. Ben-Jacob, O. Shocher, A. Tenenbaum, I. Cohen, A. Czirok and T. Vicsek, Nature 368, 46 (1994).

[10] R. Tyson, S. R. Lubkin and J. D. Murray, Proc. R. Soc. Lond. B 266, 299 (1999). 
[11] M. Matsushita, J. Wakita, H. Itoh, K. Watanabe, T. Arai, T. Matsuyama, H. Sakaguchi and M. Mimura, Physica A 274, 190 (1999).

[12] H. Meinhardt, Models of Biological Patterns Formation (Academic Press, New York, 1982).

[13] Z. Fei, B. J. Green and J. L. Hudson, J. Phys. Chem. B 103, 2178 (1999)

[14] J.-J. Perraud, A. De Wit, E. Dulos, P. De Kepper, G. Dewel and P. Borckmans, Phys. Rev. Lett. 71, 1272 (1993).

[15] A. De Wit, D. Lima, G. Dewel and P. Borckmans, Phys. Rev. E 54, 261 (1996).

[16] A. Kaminaga, V. K. Vanag and I. R. Epstein, J. Phys. Chem. 122, 174706 (2005).

[17] M. Meixner, A. De Wit, S. Bose and E. Schöll, Phys.
Rev. E 55, 6690 (1997).

[18] L. Yang and I. R. Epstein, Phys. Rev. Lett. 90, 178303 (2003).

[19] L. Yang, A. M. Zhabotinsky and I. R. Epstein, Phys. Rev. Lett. 92, 198303 (2004).

[20] L. Yang, M. Dolnik, A. M. Zhabotinsky and I. R. Epstein, J. Chem. Phys. 117, 7259 (2002).

[21] V. K. Vanag and I. R. Epstein, Phys. Rev. E 71, 066212 (2005).

[22] R. A. Barrio, C. Varea, J. L. Aragón and P. K. Maini, Bull. Math. Biol. 61, 483 (1999).

[23] J. D. Murray, Mathematical Biology II: Spatial Models and Biomedical Applications, 3rd ed. (Springer-Verlag , Berlin, 2003). 